\title{
Stock Trend Analysis and Trading Strategy
}

\author{
$\begin{array}{llll}\text { Hongxing He }^{1} & \text { Jie Chen }^{1} & \text { Huidong Jin } & \\ & \text { Shuheng Chen } & \end{array}$ \\ ${ }^{1}$ CSIRO Mathematical and Information Sciences, GPO Box 664, Canberra, ACT 2601, Australia \\ ${ }^{2}$ AI_ECON Research Center, Department of Economics, National Chengchi University, Taipei, Taiwan 11623
}

\begin{abstract}
This paper outlines a data mining approach to analysis and prediction of the trend of stock prices. The approach consists of three steps, namely partitioning, analysis and prediction. A modification of the commonly used $k$-means clustering algorithm is used to partition stock price time series data. After data partition, linear regression is used to analyse the trend within each cluster. The results of the linear regression are then used for trend prediction for windowed time series data. The approach is efficient and effective at predicting forward trends of stock prices. Using our trend prediction methodology, we propose a trading strategy TTP (Trading based on Trend Prediction). Some preliminary results of applying TTP to stock trading are reported.
\end{abstract}

Keywords: Data Mining, Clustering, $k$-means, Time Series, Stock Trading

\section{Introduction}

Trend analysis and prediction play a vital role in practical stock trading. Experienced stock traders can often predict the future trend of a stock's price based on their observations of the performance of the stock in the past. An early sign of a familiar pattern may alert a domain expert to what is likely to happen in the near future. They can then formulate their trading strategy accordingly.

The search for and matching of similar patterns have been studied extensively on time series analysis $[1,2]$. Patterns in long time series data repeat themselves due to seasonality or other unknown underlying reasons. Early detection of patterns similar to those that have occurred in the past can readily provide information on what will follow. This informa- tion will be able to help decision-making on the trading strategy in stock market trading practice. In this paper, we report an approach to predict the trend of the stock prices and apply it to stock trading practice.

\section{Time Series Data Preparation}

We create training data by sliding a fixed-length time window from time $t_{b}$ to $t_{e}$. The following $N=t_{e}-t_{b}$ time series are created with a given window length $w_{\text {tr }}$.

$$
\begin{gathered}
s_{1}: p_{1}, p_{2}, \ldots, p_{w_{\mathrm{tr}}} \\
s_{2}: p_{2}, p_{3}, \ldots, p_{w_{\mathrm{tr}}+1} \\
\ldots \\
s_{N}: p_{N}, p_{N+1}, \ldots, p_{w_{\mathrm{tr}}+N-1}
\end{gathered}
$$

where $p_{i}\left(i=1,2, \cdots, w_{\mathrm{tr}}+N-1\right)$ are stock prices at time $i$. We therefore create an $N$ by $w_{\text {tr }}$ matrix or a data set with $N$ data records and $w_{\text {tr }}$ attributes. Note that all attributes take continuous values and conventional data mining methods can be applied directly $[3,4]$.

For the test data, we use another window of length $w_{\mathrm{te}}<w_{\mathrm{tr}}$. Each training windowed series is then divided into two parts. The first part has the same length as the test data. The second part of length $w_{\mathrm{lm}}=w_{\mathrm{tr}}-w_{\mathrm{te}}$ is used to decide the classification of a cluster. All windowed time series are properly normalised. Figure 1 gives a schematic view of a windowed time series.

\section{Methodology for Trend Analy- sis}

Our data mining approach consists of the following steps. 


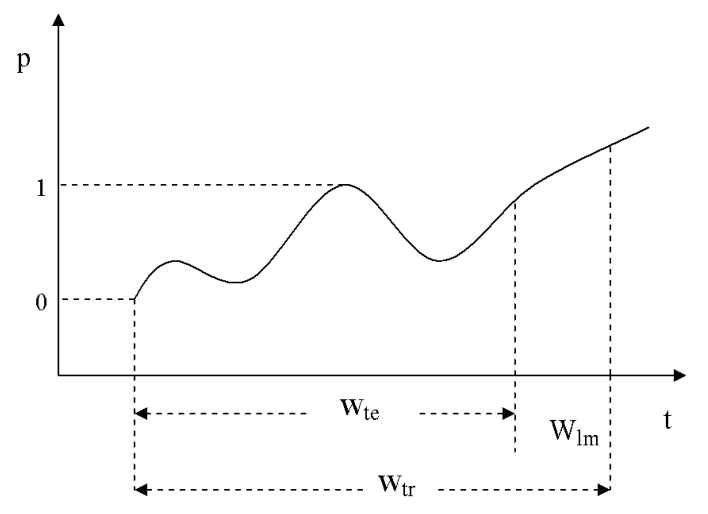

Figure 1: Schematic view of windowed time series and normalisation

1. Initialisation.

- Select window lengths $w_{\mathrm{tr}}$ and $w_{\mathrm{te}}$ for training and test data respectively.

- Select a test period.

For example, if we test the method for year 1999-2000, then the test period starts from the first trading day of 1999 to the last trading day of 2000 .

- Select training period.

The training sample will start from $w_{\text {tr }}$ days before the first trading day of year 1989 and end on the last trading day of year 1998 in the aforementioned example.

2. Data Mining.

- Create $N$ training series of window length $w_{\mathrm{tr}}$ from training period.

- Normalise each series individually such that the first $w_{\text {te }}$ values of the series fall between 0 and 1 .

- Partition the training data into $k$ clusters, which are represented by their cluster centers. We use the $k$-means clustering to group the training data based on attributes into $k$ groups [5]. $k>1$ is a pre-specified integer number.

- Classify all the clusters into two distinct classes using a linear regression model [6]. A model is built based on the last $w_{\mathrm{lm}}$ values of each cluster center. Class "UP" is labeled if the gradient is positive and "DOWN" otherwise.

3. Test models on test data.

- Form a test series dataset with the window length $w_{\text {te }}$. Normalise them individually. Consequently, values will fall between 0 and 1 .

- Assign a cluster label $c_{i}=j$ to time series $i$ in test data such that cluster $j(j=$ $1,2, \cdots, k)$ has the smallest Euclidean distance to the normalised series $i$.

- Assign the class ("UP" or "DOWN") of cluster $j$ to time series $i$, where time series $i$ has cluster label $j$.

- Calculate returns for a selected trading strategy.

\section{Trading Strategies}

In this section we introduce two trading strategies. The first strategy is naive trading, where future trend is not taken into consideration. The second is same as the first except that the future trend prediction is used in trading decision.

Naive Trading (NT) We call our trading strategy "naive trading" because it is simplistic. In NT, we buy the stock if we are not holding a share and the purchase cost is lower than the value at which we sold previously. By the same token, we sell the stock if we hold a share and we can make profit from that sale of any margin. Thus, short-selling is included. That is, we sell the stock if the value received exceeds the value at which we bought previously.

Trading based on Trend Prediction (TTP) TTP is a slight variation of NT. The only difference is that we consider the forward trend of the stock price. We sell the share only if the trend prediction is downward.

\section{Experimental Results}

In this section we report some preliminary results. In order to compare our trading strategy with other existing strategies we follow [7] closely. In order to com- 
pare our trading strategies with other existing strategies, we test them on one time period, namely for year 1999-2000. The corresponding training period is 1989-1998 (ten years). The comparison is made with [7]. To facilitate the comparison, stock indexes from five countries are used in the paper.

Tables 1 lists the return from NT, TTP, GP (Genetic Programming) and twenty one practical trading strategies for selected countries in the test time period. The values listed are the investment returns as fraction (for example, 0.1778 in Table 1 means that the return is $17.78 \%$ ). For more details please refer to [7]. The B\&H refers to buy and hold strategy.

We have the following observations based on the results presented in Table 1 .

1. TTP's performance exceeds NT's performance in most countries. This clearly indicates that the trend prediction is able to find the correct trend in some cases. The trading strategy considering the price trend does improve the trading performance.

2. As shown in Table 1 for the time period 1999 2000, TTP has the best performance for US and Singapore in comparison with GPs, i.e., GP 1 and 2 , and the twenty one practical trading strategies. For UK, NT, which is slightly better than TTP, performs the best. While all the twenty one practical trading strategies get negative or a slight positive return, TTP is able to produce significant positive returns for the time period 1999-2000. For Canada, GPs perform best, which is followed by $\mathrm{B} \& \mathrm{H}$. TTP gives a slight positive return while most of the twenty one practical strategies get negative returns. For Taiwan, the GPs perform much better than all the other trading strategies. However TTP is able to exceed $\mathrm{B} \& \mathrm{H}$ and most of the twenty one practical strategies.

\section{Conclusions and Future Work}

We have applied a data mining approach to analyse and predict the trend of the stock price and applied it in real stock trading practice. Results have shown that the proposed methodology improves the trading performance over some existing strategies in some cases. While the methodology developed in the work can correctly predict the trend of stock prices for some countries, it is not able to predict well for all. The stock price is very volatile in nature. The proposed trend prediction approach certainly has its limitations. The following future work may improve the performance of the method.

1. A simple decision on classification of clusters is made using the linear regression model in the present work. We can further improve the accuracy of the trend prediction by using fuzzy or probabilistic decision systems in the future.

2. Improve the computation efficiency by using sophisticated and scalable clustering techniques, such as $[4,8]$.

3. Introducing scale change to pattern matching can discover similar patterns with different time scales.

4. Combine our method with other techniques, such as GP, for better and more sophisticated trading strategies.

\section{Acknowledgements}

The authors acknowledge Damien McAullay and Arun Vishwanath for their assistance in the preparation of the paper.

\section{References}

[1] X. Ge. Pattern matching financial time series data. Project Report ICS 278, UC Irvine, 1998.

[2] E. Keogh and P. Smyth. A probabilistic approach to fast pattern matching in time series databeses. In Proceedings of KDD'97, pages 24-30, Newport beach, CA, USA, 1997.

[3] J. Han and M. Kamber. Data Mining: Concepts and Techniques. Morgan Kaufmann Publishers, San Francisco, CA, USA, 2001.

[4] H.-D. Jin, M.-L. Wong, and K.-S. Leung. Scalable model-based clustering for large databases based on data summarization. IEEE Transactions on Pattern Analysis and Machine Intelligence, 27(11):1710-1719, Nov. 2005. 
Table 1: The Total Return of Stock Trading for 1999-2000 in comparison with GP and 21 practical trading strategies

\begin{tabular}{|l|l|l|l|l|l|}
\hline Rule & USA & UK & Canada & Taiwan & Singapore \\
\hline B \& H & 0.0636 & 0.0478 & 0.3495 & -0.2366 & 0.3625 \\
\hline GP 1 & 0.0655 & 0.0459 & 0.3660 & 0.1620 & 0.1461 \\
\hline GP 2 & 0.0685 & 0.0444 & 0.3414 & 0.5265 & 0.1620 \\
\hline TTP & 0.1778 & 0.1524 & 0.0541 & -0.22 & 0.4654 \\
\hline NT & 0.0786 & 0.1560 & 0.0207 & -0.1480 & 0.0524 \\
\hline 1 & -1.1173 & -1.2855 & -1.8943 & -1.5102 & -1.0679 \\
2 & 0.0292 & -0.5265 & -0.9935 & -0.8737 & -0.8182 \\
3 & -0.1640 & -0.6941 & -0.2494 & -0.3338 & -0.7028 \\
4 & -0.9865 & -0.8252 & -0.1182 & -0.7371 & -0.5123 \\
5 & -0.0896 & -0.3062 & -0.9872 & -0.2571 & -0.6288 \\
6 & -0.7176 & -0.6335 & -0.0440 & 0.0048 & -0.7599 \\
7 & -1.1736 & -1.7050 & -2.1544 & -1.1646 & -1.9132 \\
8 & -1.2402 & -1.3594 & -2.1444 & -0.7130 & -0.8391 \\
9 & -1.3883 & -1.0738 & -1.6657 & -1.0748 & -0.7450 \\
10 & -1.6532 & -1.4603 & -1.5322 & -1.0678 & -0.4226 \\
11 & -1.0941 & -0.5934 & -1.4946 & -0.3628 & -0.9329 \\
12 & -1.4735 & -1.2046 & -2.6474 & -1.5254 & -1.6464 \\
13 & -0.9116 & -0.7762 & -0.1522 & -0.6863 & -0.3210 \\
14 & -0.2477 & -0.2666 & -0.9692 & -0.2258 & -0.5817 \\
15 & -0.6658 & -0.5571 & 0.0019 & 0.0218 & -0.7405 \\
16 & -0.7576 & -0.9016 & -0.1671 & -0.4350 & -0.0302 \\
17 & -0.1607 & 0.0126 & -1.0631 & 0.3375 & -0.5044 \\
18 & -0.4397 & -0.6185 & -0.0055 & 0.1213 & -0.4336 \\
19 & -0.4240 & -0.7951 & -0.0942 & -0.1480 & -0.1412 \\
20 & 0.1419 & -0.0474 & -1.0680 & -0.5793 & -0.5628 \\
21 & -0.4195 & -0.6143 & 0.0827 & 0.2087 & -0.5644 \\
\hline
\end{tabular}

[5] J. B. MacQueen. Some methods for classification and analysis of multivariate observations. In Proceedings of 5-th Berkeley Symposium on Mathematical Statistics and Probability, pages 281297, Berkeley, University of California, 1967.

[6] J. M. Chambers and T.J. Hastie, editors. Statistical Models in S, chapter Linear Models. Wadsworth \& Brooks/Cole, 1992.

[7] S. H. Chen, T. W. Kuo, and K. M. Hsu. Handbook of Financial Engineering, chapter Genetic Programming and Financial Trading: How Much about "What we Know"? Kluwer Academic Publishers, 2006.

[8] H.-D. Jin, K.-S. Leung, M.-L. Wong, and Z.B. Xu. Scalable model-based cluster analysis using clustering features. Pattern Recognition, 38(5):637-649, May 2005. 